\title{
A Case of Prenatal Presentation with Double Aortic Arch
}

Nobuko Shiono ${ }^{1}$, Kohta Takei², Takahiro Yamada ${ }^{2}$, Tsuyoshi Tachibana ${ }^{3}$, Kazutoshi Cho ${ }^{2^{*}}$ and Hisanori Minakami ${ }^{2}$

${ }^{1}$ Department of Neonatology, Perinatal Medical Center, Sapporo City General Hospital, Sapporo, Japan

${ }^{2}$ Department of Perinatal Medicine, Hokkaido University Hospital, Sapporo, Japan

${ }^{3}$ Department of Cardiovascular Surgery, Hokkaido University Graduate School of Medicine, Sapporo, Japan

*Corresponding author: Kazutoshi Cho, Department of Perinatal Medicine, Hokkaido University Hospital, Sapporo, Japan, Tel: 8111706584; E-mail: chotarou@med.hokudai.ac.jp

Rec date: August 21, 2015; Acc date: August 31, 2015; Pub date: September 11, 2015

Copyright: (C) 2015 Shiono N. This is an open-access article distributed under the terms of the Creative Commons Attribution License, which permits unrestricted use, distribution, and reproduction in any medium, provided the original author and source are credited.

\begin{abstract}
Double aortic arch (DAA) is a form of vascular ring found in approximately 1 in 2000-4000 pregnancies on fetal echocardiography. In some cases of DAA, respiratory compromise occurs during infancy due to compression of the trachea, which is completely encircled by the right aortic arch (RAA) and left aortic arch (LAA). RAA and left arterial duct were initially seen on screening fetal echocardiography in a 36-year-old Japanese woman at gestational week (GW) 23. The fetus was later found to have DAA by detailed echocardiography at GW 26 . The female neonate born at GW 38 weighing $2894 \mathrm{~g}$ with 1- and 5-minute Apgar scores of 8 and 9, respectively, developed tachypnea, stridor, and wheezing soon after birth, and underwent successful surgical correction 10 hours after birth. Thus, prenatal diagnosis of DAA aided in early recovery in this case.
\end{abstract}

Keywords Double aortic arch; Echocardiography; Prenatal diagnosis; Vascular ring

\section{Introduction}

Left and right aortic arch refers to the position of the aortic arch in relation to the trachea. Normal embryological development of the arch is from the primitive pharyngeal arch system. The normal left aortic arch (LAA), descending on the left side of the trachea, is formed from the left fourth arch and the left dorsal aorta with regression of a segment of the embryological right aortic arch (RAA) [1].

Double aortic arch (DAA) is a form of vascular ring in which the trachea and esophagus are completely encircled by the RAA and LAA. This condition causes compression of the trachea and esophagus, leading to various symptoms, including respiratory obstruction, dysphagia, chronic wheezing, vomiting, and aspiration [2,3]. A late diagnosis is common, and this may aggravate tracheal damage due to persistent compression [4]. DAA is found in approximately 1 in 20004000 pregnancies on fetal echocardiography [1,5] and in $1.5 \%$ of abnormal fetal echocardiography examinations [6]. To our knowledge, there have been only a few reports on prenatal diagnosis of DAA [1,5-8]. We recently encountered a case of DAA that was diagnosed prenatally.

\section{Case Report}

The Hokkaido University Hospital Institutional Review Board approved this study and the patient provided signed informed consent.

A 36-year-old Japanese woman underwent screening echocardiography of spatiotemporal image correlation (STIC) $[9,10]$ including both grayscale and color Doppler studies at gestational week (GW) 23. The images revealed RAA and left arterial duct, but not LAA (Figure 1). Subsequent repeat echocardiographic examinations at GW $26,29,32$, and 38 consistently suggested the presence of DAA with the trachea encircled by the RAA and LAA (Figure 2) and normal intracardiac anatomy. A female neonate was born by repeat cesarean section, weighing $2894 \mathrm{~g}$ with 1- and 5-minute Apgar scores of 8 and 9, respectively. Within 10 minutes after birth, she exhibited respiratory difficulty, including tachypnea, persistent stridor, and wheezing, and required inhaled oxygen with continuous positive airway pressure. Postnatal computed tomography (CT) angiography of the thorax confirmed the diagnosis of DAA with mild narrowing of the midtrachea in this patient (Figure 3). Surgical treatment, including division of the LAA and left arterial duct approached via the side of the arterial duct, was performed 10 hours after birth. The postoperative course was uneventful, and the infant left hospital at age 15 days. The infant was free from any symptoms when last seen at 1 month old.
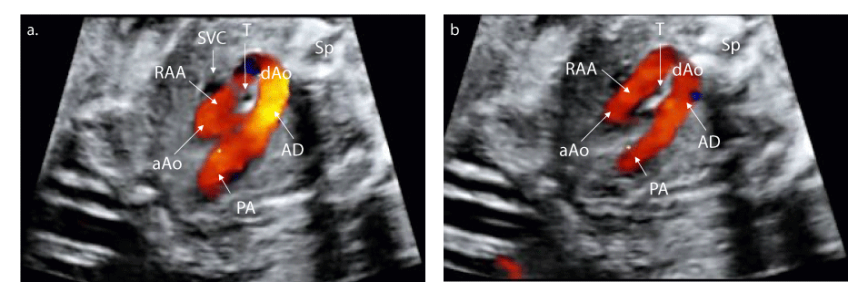

Figure 1: Screening echocardiography at GW 23 with spatiotemporal image correlation (STIC) system. The images show the presence of right aortic arch (RAA) in three-vessel tracheal view. a) The transverse aorta lies to the right of the trachea (T). Left arterial duct $(\mathrm{AD})$ runs posteriorly and is continuous with the pulmonary artery (PA). The superior vena cava (SVC) lies to the right of the aortic arch. b) The RAA and AD traverse posteriorly forming a classic " $U$ " shape. No left aortic arch is detected. aAo, ascending aorta; dAo, descending aorta; Sp, spine. 


\section{Discussion}

An early surgical intervention based on prenatal diagnosis of DAA helped this patient to recovery early from respiratory difficulties due to compression of the trachea by the DAA. As seen in this patient, DAA is likely to present as an isolated RAA initially on prenatal echocardiography $[5,6]$. RAA alone was initially found in two of three [5] and one of two cases with DAA [6] reported previously.

Respiratory symptoms can develop in infants with either an isolated RAA or DAA [11]. However, respiratory symptoms develop in most patients with prenatal diagnosis of DAA 1 and are more severe in infants with DAA than in those with an isolated RAA [11]. Symptoms of DAA mimic "asthma-like" respiratory symptoms during infancy and the diagnosis of DAA is often delayed in infants without prenatal suspicion or diagnosis of DAA [4]. More than $70 \%$ of patients with delayed surgical repair suffer from long-lasting respiratory problems associated with compression-induced maldevelopment of the trachea [12]. The surgical approach for DAA is effective, postoperative complications are infrequent, and outcomes are excellent after repair of DAA with low mortality rates $[12,13]$ as seen in this patient. Therefore, prenatal diagnosis or suspicion of DAA may be clinically important.
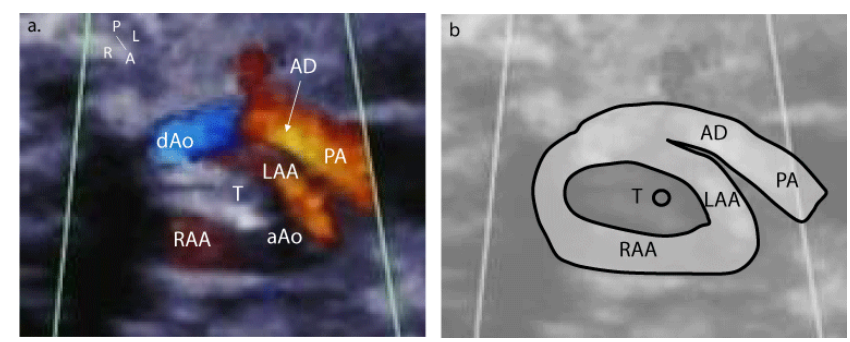

Figure 2: Echocardiography at GW 29 performed by a pediatric cardiologist. a) The image shows the features of a double aortic arch with the right aortic arch (RAA) and left aortic arch (LAA) encircling the trachea $(T)$ in three-vessel tracheal view. b) The two aortic arches, main pulmonary artery (PA), and arterial duct (AD) together form a vascular complex with a figure "9"configuration around the trachea $(\mathrm{T})$. aAo, ascending aorta; dAo, descending aorta; $\mathrm{P}$, posterior; $\mathrm{A}$, anterior; $\mathrm{L}$, left; $\mathrm{R}$, right.

DAA is found in approximately $7.0 \%$ of cases with RAA $(6.1 \%$ [6/98] Miranda et al. [1] and 7.7\% [4/52] Razon et al. [5]). Further examination for detection of LAA is recommended in cases with RAA $[6,7]$. A figure " 9 " formed by the two aortic arches, main pulmonary arterial trunk, and arterial duct around the trachea may be seen on fetal upper mediastinum transverse planes in cases with DAA [14], as seen in the present case. In addition, chromosomal analysis is recommended in cases with RAA because $15 \%$ of affected infants have chromosomal aberrations, half of which involve 22q11.2 microdeletion [1].

STIC is a technology that allows automatic storage of volume data sets of fetal hearts in a four-dimensional (4D) cineloop sequence, thus reducing the operator skill factor required and allowing other observers to review the data offline $[9,10]$. The advantages of STIC, such as slow-motion imaging and the capability of analyzing the fetal heart without fetal movements, allowed us to better assess the anomaly in this case. The aortic arch and trachea were imaged in transverse view of the fetal upper thorax (three-vessel and tracheal view) on prenatal echocardiography. As RAA is usually a large main aortic arch and LAA is relatively small in 70\% of cases with DAA [15] and as the transverse aorta lies to the right of the trachea, the RAA can be readily identified in a transverse view of the fetal upper thorax.

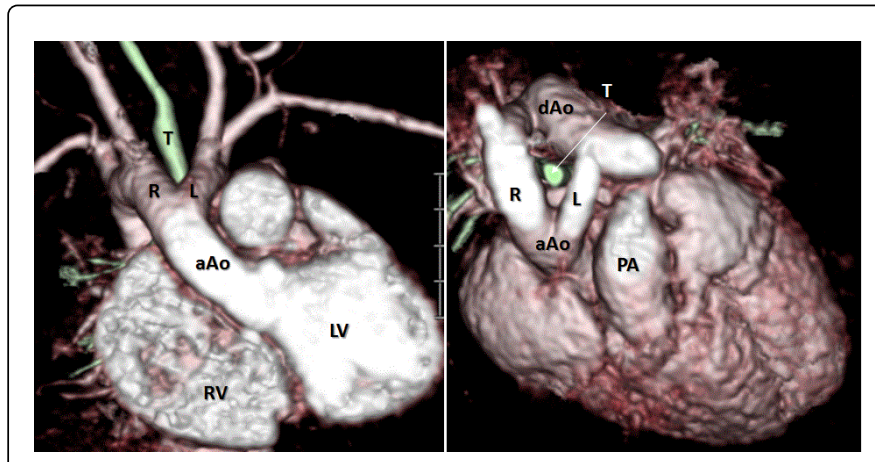

Figure 3: Postnatal CT angiography performed 7 hours after birth. The large right aortic arch (R) and small left aortic arch (L) encircle the trachea (T) forming a vascular ring. Mild narrowing of the midtrachea $(\mathrm{T})$ is also demonstrated. $\mathrm{LV}$, left ventricle; RV, right ventricle; aAo, ascending aorta; dAo, descending aorta; PA, pulmonary artery.

A patient with prenatal diagnosis of DAA was presented. The patient exhibited respiratory problems soon after birth, but prompt surgical decompression of the trachea contributed to early resolution of these problems derived from DAA. Our experience emphasized that RAA is a key finding leading to prenatal diagnosis of DAA.

\section{References}

1. Miranda JO, Callaghan N, Miller O, Simpson J, Sharland G (2014) Right aortic arch diagnosed antenatally: associations and outcome in 98 fetuses. Heart 100: 54-59.

2. Turner A, Gavel G, Coutts J (2005) Vascular rings--presentation, investigation and outcome. Eur J Pediatr 164: 266-270.

3. Valletta EA, Pregarz M, Bergamo-Andreis IA, Boner AL (1997) Tracheoesophageal compression due to congenital vascular anomalies (vascular rings). Pediatr Pulmonol 24: 93-105.

4. Ullmann N, Menchini L, Salerno T, Tomà P, Cutrera R (2014) Late diagnosis of double aortic arch: consequences on long-term follow-up. Pediatr Pulmonol 49: E75-77.

5. Razon Y, Berant M, Fogelman R, Amir G, Birk E2 (2014) Prenatal diagnosis and outcome of right aortic arch without significant intracardiac anomaly. J Am Soc Echocardiogr 27: 1352-1358.

6. Seo HK, Je HG, Kang IS, Lim KA (2010) Prenatal double aortic arch presenting with a right aortic arch and an anomalous artery arising from the ascending aorta. Int J Cardiovasc Imaging 1: 165-168.

7. Hunter L, Callaghan N, Patel K, Rinaldi L, Bellsham-Revell H, et al. (2015) Prenatal echocardiographic diagnosis of double aortic arch. Ultrasound Obstet Gynecol 45: 483-485.

8. Yao WM, Wang JM, Huang HF, Ye YH (2010) Prenatal diagnosis of double aortic arch and D-transposition of the great arteries: a case report. Prenat Diagn 30: 382-383.

9. Viñals F, Poblete P, Giuliano A (2003) Spatio-temporal image correlation (STIC): a new tool for the prenatal screening of congenital heart defects. Ultrasound Obstet Gynecol 22: 388-394.

10. DeVore GR, Falkensammer P, Sklansky MS, Platt LD (2003) Spatiotemporal image correlation (STIC): new technology for evaluation of the fetal heart. Ultrasound Obstet Gynecol 22: 380-387. 
Citation: Shiono, Takei, Yamada, Tachibana, Cho Minakami, et al. (2015) A Case of Prenatal Presentation with Double Aortic Arch. J Neonatal Biol 4: 194. doi:10.4172/2167-0897.1000194

Page 3 of 3

11. Roehr CC, Wilitzki S, Opgen-Rhein B, Kalache K, Proquitté H, et al (2011) Early lung function testing in infants with aortic arch anomalies identifies patients at risk for airway obstruction. PLoS One 6: e24903.

12. Fleck RJ, Pacharn P, Fricke BL, Ziegler MA, Cotton RT, et al. (2002) Imaging findings in pediatric patients with persistent airway symptoms after surgery for double aortic arch. AJR Am J Roentgenol 178: 1275-1279.

13. Bertolini A, Pelizza A, Panizzon G, Moretti R, Bava CL, et al. (1987) Vascular rings and slings. Diagnosis and surgical treatment of 49 patients. J Cardiovasc Surg (Torino) 28: 301-312.
14. Yoo SJ, Min JY, Lee YH, Roman K, Jaeggi E, et al. (2003) Fetal sonographic diagnosis of aortic arch anomalies. Ultrasound Obstet Gynecol 22: 535-546.

15. Alsenaidi K, Gurofsky R, Karamlou T, Williams WG, McCrindle BW (2006) Management and outcomes of double aortic arch in 81 patients. Pediatrics 118: e1336-1341. 